University of Wollongong

Research Online

Faculty of Engineering - Papers (Archive)

Faculty of Engineering and Information

Sciences

$1-1-2008$

\title{
Emission of terahertz-frequency electromagnetic radiation from indium phosphide under excitation by short pulses of near-infrared radiation
}

Stuart Hargreaves

University of Wollongong, sh64@uow.edu.au

Roger A. Lewis

University of Wollongong, roger@uow.edu.au

Follow this and additional works at: https://ro.uow.edu.au/engpapers

Part of the Engineering Commons

https://ro.uow.edu.au/engpapers/4007

\section{Recommended Citation}

Hargreaves, Stuart and Lewis, Roger A.: Emission of terahertz-frequency electromagnetic radiation from indium phosphide under excitation by short pulses of near-infrared radiation 2008, 1-2.

https://ro.uow.edu.au/engpapers/4007

Research Online is the open access institutional repository for the University of Wollongong. For further information contact the UOW Library: research-pubs@uow.edu.au 


\title{
Emission of terahertz-frequency electromagnetic radiation from indium phosphide under excitation by short pulses of near-infrared radiation
}

\author{
S. Hargreaves and R. A. Lewis, \\ Institute for Superconducting and Electronic Materials, University of Wollongong, Wollongong, NSW
} 2522, Australia

\begin{abstract}
We have examined the emission of terahertzfrequency radiation from Indium Phosphide (InP) under ultrashort pulses of near-infrared radiation. We have investigated nominally undoped, as well as p-doped and n-doped material. Compared to the emitter Indium Arsenide (InAs) we find that the total THz field from undoped InP is an order of magnitude less, but with the strength shifted to higher frequencies.
\end{abstract}

\section{INTRODUCTION AND BACKGROUND}

A number of distinct physical mechanisms give rise to $\mathrm{THz}$ radiation when semiconductors are illuminated with above-band gap radiation. These include optical rectification, as exhibited by Zinc Telluride ( $\mathrm{ZnTe}$ ) and photoconduction, as exhibited by Gallium Arsenide (GaAs) on which suitable electrodes have been fabricated ${ }^{1}$. A third class of emitters operates according to surface-field effects; the strongest of these to date appears to be InAs ${ }^{2}$. In this work we examine another III-V semiconductor alloy, InP, as a surface-field emitter. We have examined both $\mathrm{p}$-type $\mathrm{t}^{3}$ and $\mathrm{n}$-type $\mathrm{InP}^{4}$, as well as nominally undoped material. It is the results for the undoped material which will be presented in this paper.

\section{EXPERIMENTAL DETAILS}

We employed a conventional time-domain spectroscopy (TDS) configuration based around a 12-fs laser of center frequency $790 \mathrm{~nm}$, and a repetition rate of $75 \mathrm{MHz}$. The infrared optical power incident at the sample was approximately $300 \mathrm{~mW}$.

Detection of the radiation is by the electro-optic effect in a ZnTe wafer illuminated by a probe pulse of variable delay. $\mathrm{THz}$ radiation induces birefringence in the probe pulse at the $\mathrm{ZnTe}$ crystal, and the final signal is the differential output of a photodiode pair.

Measurements were conducted at atmospheric pressure in the presence of water vapor - both for simplicity and because the well-known rotational absorption lines of water vapor provide convenient calibration and reference points.

Four nominally undoped InP samples were tested, as was a nominally undoped InAs reference sample. All samples were tested in a 90 degree reflection geometry, i.e. with the front surface of the sample at 45 degrees to both the incident and reflected beams.



Fig. 1. Simplified diagram of the system in a 90 degree reflection configuration for the experiment (not to scale). The dotted line marks the near-infrared laser pulse, and the solid grey line marks the terahertz beam path.

\section{RESULTS}

Figure 2 depicts the time-domain data for both the strongest InP test emitter and a reference InAs emitter under identical excitation. It will be observed that the InP field signal is of the order of one magnitude less than the InAs field signal and consequently the intensity about two orders of magnitude less. To put this result in context, it is still considerably stronger than the optical-rectification signal generated in ZnTe in the same system, but much less than the signal obtained from suitably biased photoconductive emitters ${ }^{1}$.

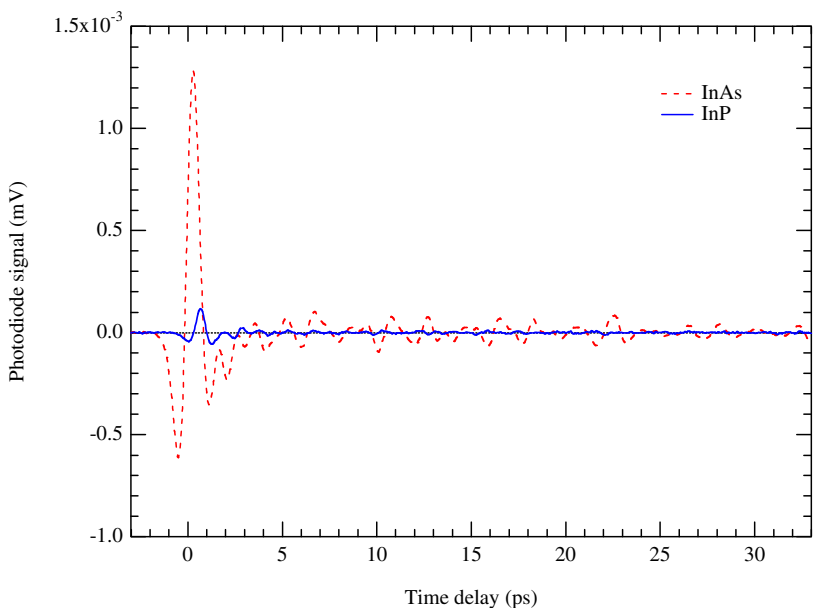

Fig. 2. Time-domain data for two semiconductor emitters, InAs (red dashed curve) and InP (blue full curve).

Figure 3 illustrates the frequency-domain data for InP, with 
InAs data given for comparison. In Fig. 2 the left axis applies to InP and the right axis applies to InAs. Both axes ranges have been set so that the maximum value of the data is at the top of the figure and the bottom of the figure is five orders of magnitude less.

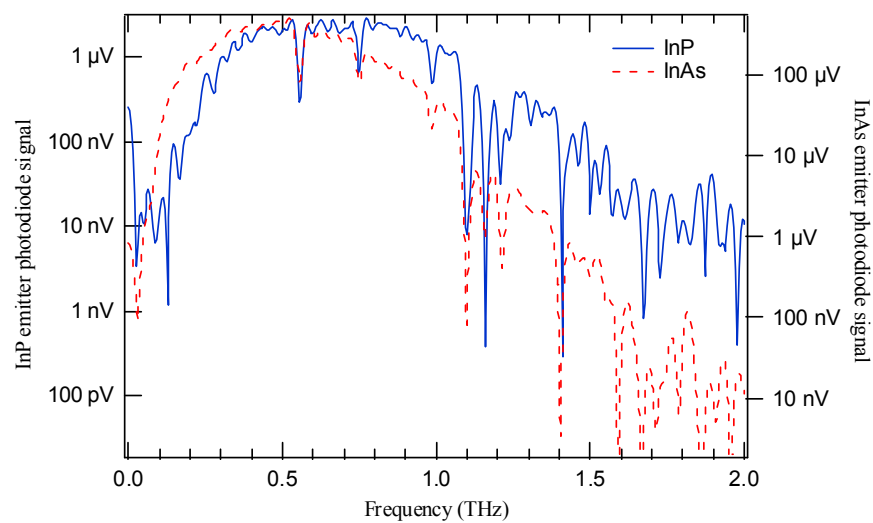

Fig. 3. Frequency-domain data for two semiconductor emitters, InAs (red dashed curve) and InP (blue full curve).

For both InP and InAs, water vapor lines at $0.557 \mathrm{THz}$, $0.753 \mathrm{THz}, 0.989 \mathrm{THz}, 1.098 \mathrm{THz}, 1.164 \mathrm{THz}, 1.226 \mathrm{THz}$ and $1.412 \mathrm{THz}^{6}$ were clearly evident. Above approximately 1.5 $\mathrm{THz}$, however, the observed signal was too weak in both samples to identify any further absorption lines.

While it is clear that the intensity from the InP emitter is much less than that from the InAs emitter, the signal-to-noise ratios are comparable. Moreover, the InP emitter has a relatively much stronger signal above $0.6 \mathrm{THz}$. This may make it the preferred emitter over InAs in circumstances where these frequencies are of most interest.

The results from the different InP samples were in reasonable agreement - the weakest of the four samples had a peak field intensity $30 \%$ lower than that of the strongest of the four samples.

\section{CONCLUSION}

Although we have found the peak intensity of the surfacefield emission from InP to be about an order of magnitude less than that of InAs, above $0.6 \mathrm{THz}$ the InP sample exhibits a proportionally stronger response. It is, however, not clear to what extent the high-frequency roll-off is an inherent property of the samples. If most of the roll-off is caused elsewhere in the system, the difference in high-frequency response between the two samples may be even more pronounced. Attempting to isolate other potential causes of the high-frequency roll-off may yield additional information.

If time-domain terahertz measurements require spectral information at high frequencies, our results indicate that InP may be a more useful choice of emitter than InAs.

\section{REFERENCES}

[1] S. Hargreaves, R. A. Lewis, M. Henini, "Semiconductor terahertz emitters", Proceedings of SPIE 6798-34 (2007).
[2] R. A. Lewis, M. L. Smith, R. Mendis, R. E. M. Vickers, "THz generation in InAs", Physica B: Physics of Condensed Matter 376-377, 618-621 (2006).

[3] R. A. Lewis, Y.-J. Wang, "Magneto-optical far-infrared absorption spectroscopy of the hole states of indium phosphide", Physical Review B 71, 115211 (2005).

[4] R. A. Lewis, P. E. Simmonds, Y.-J. Wang, "Magnetopolaron interactions in n-type indium phosphide", Physical Review B 72, 245207 (2005).

[5] S. Hargreaves and R. A. Lewis, "Terahertz imaging: materials and methods", Journal of Materials Science: Materials in Electronics 18, S299-S303 (2007).

[6] J-M. Flaud, C. Camy-Peyret, and R. A. Toth, "International Tables of Selected Constants" (Pergamon, Oxford, 1987) 